\title{
Analysis of Rigid Extended Object Co-Manipulation by Human Dyads: Lateral Movement Characterization
}

\author{
Erich A. Mielke Eric C. Townsend Marc D. Killpack
}

\begin{abstract}
For co-manipulation involving humans and robots, robot controllers that are based on human-human behavior should allow more comfortable and coordinated movement between the human-robot dyad. In this paper, we describe an experiment between human-human dyads where we recorded the force and motion data as leader-follower dyads moved in translation and rotation. The force/motion data was then analyzed for patterns found during lateral translation only. For extended objects, lateral translation and in-place rotation are ambiguous, but this paper determines a way to characterize lateral translation triggers for future use in human-robot interaction. The study has 4 main results. First, interaction forces are non-negligible and are necessary for co-manipulation. Second, minimum-jerk trajectories are found in the lateral direction only for lateral movement. Third, the beginning of a lateral movement is characterized by distinct force triggers by the leader. Fourth, there are different metrics that can be calculated to determine which dyads moved most effectively in the lateral direction.
\end{abstract}

\section{INTRODUCTION}

The concept of humans and robots collaboratively working to accomplish useful tasks is currently motivating a large amount of robotics research. Physical Human-Robot Interaction (pHRI) is an area of robotics that can especially benefit from the combined strengths of a human-robot team: strength and execution from the robot and intelligence and planning from the human. This is particularly true of comanipulation tasks where a human and a robot physically manipulate the same object simultaneously. Co-manipulation can include complex translational and rotational tasks, such as moving a table, couch, or other extended, rigid objects. These objects are heavy or unwieldy, and necessitate two or more people to carry them. A robot capable of replacing a human in these teams would help greatly in situations like search and rescue where current high-payload robots are too heavy and dangerous to relocate and operate. Robots that can physically interact with a human could help lift and remove rubble from disaster areas or take a victim on a stretcher to safety. These robots would allow fewer people to complete the same amount of work, or for more teams to operate and reach more people in need of help. Other applications include using robots to help load and unload moving vans, using robots to help move objects around warehouses, and any other co-manipulation applications where human-human teams are currently used (see Fig. 1).

An important characteristic of these situations is uncertainty in the task. Often a task is poorly defined for one or both partners of a dyad, and a controller needs to be able to adapt to disturbances and trajectory changes. We have found that ambiguity can exist when tasks include manip-

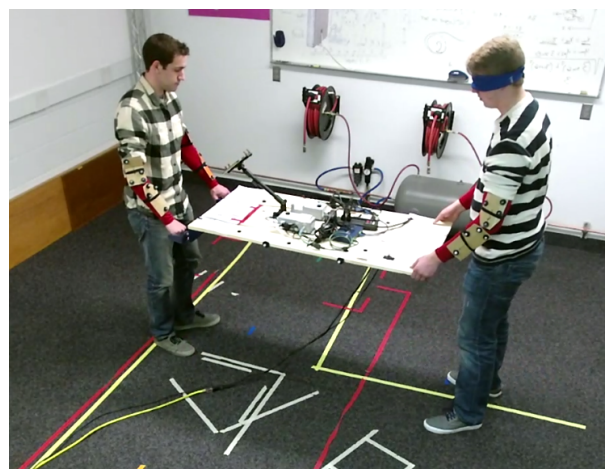

Fig. 1: A dyad, one blind follower and one sighted leader, performs a co-manipulation task

ulating an extended object that may need to be translated, rotated, or both. When an extended object is included in co-manipulation tasks, forces applied in a lateral direction could indicate either intent to translate laterally, or intent to rotate the object in the plane, which will be referred to as the rotation-translation problem. In order to be effective, a pHRI controller for co-manipulation of extended objects must be able to distinguish between rotation and translation intent.

While the past work on co-manipulation outlined in Section II shows that collaboration through force is applicable to some tasks, it is not clear that the algorithms and intentestimators developed will work in less-defined scenarios. In order for a robot to work with humans for co-manipulation, it needs to be able to respond in complex situations involving movement of the object in 6 dimensions, 3 translational and 3 rotational. Six degrees of freedom is for a rigid object only and manipulation of any non-rigid object will only increase complexity. These types of movement involve whole-body motion and bi-manual manipulation by the participants, rather than planar arm movements only. Our eventual goal is to create a controller that can be used in a human-robot dyad that is able to move in 6 DOF based on intent provided by the human. To understand how to design control methods for a robot in a human-robot co-manipulation team, our approach is to characterize the movements and forces produced by human-human dyads for a variety of tasks in order to later apply similar principles to a generalized robot controller for co-manipulation. We also expect that the principles presented in this paper on human-human co-manipulation could inform controller design for robot-robot co-manipulation in terms of stability and force control principles for efficient operation between two autonomous agents. 
The main contributions of this paper are as follows

1) Unique co-manipulation data from trials where humanhuman dyads moved a rigid table together (described in full in Section III)

2) Observations on lateral movements from comanipulation study, which include the following:

- Interaction forces are not minimized

- Lateral trajectories resemble minimum-jerk trajectories

- Lateral movements are triggered by a specific interaction force sequence

- Dyads that performed well for lateral-translation movements minimized angular velocity about the superior axis and minimized deviation from a minimum-jerk trajectory

The paper is organized as follows. Section II explores relevant literature on human-robot co-manipulation tasks. The experiment described in our contributions is explained in fulldepth in Section III, including describing the equipment used, describing each task in detail, and describing the participants of the study. Section IV explores the main observations from the study. Lastly, Section V is the conclusion and describes future work.

\section{RELATED WORK}

\section{A. State-of-the-Art Controllers}

Researchers have been studying pHRI co-manipulation for many years. While a majority of the research done has been for Cartesian motion, some people have studied the rotationtranslation problem. Thobbi et al. implemented a version of a controller for rotation-translation, although human intent was captured using motion capture, limiting its applicability [1]. Bussy et al. developed a proactive controller for a twoarmed mobile robot [2] [3]. Their model was based on an experiment with human-human dyad that manipulated an extended object data in $1 \mathrm{DOF}$ (anterior/posterior movement). Their model was a finite state machine, where each state was triggered by a force applied, and then passed through a trajectory-referenced admittance controller to determine the desired state. While this approach was successful in the translation aspect, it did not address the rotational part of the problem. Karayiannidis et al. developed a model to switch between rotational and translational movement for a one-armed stationary robot [4]. In their experiments, a human partner was able to move the robot in translation and rotation as desired. However, their experiments were done for single-arm manipulation and they approached the rotation-translation problem by manually setting values for parameters.

Many other experiments have been performed which show considerable promise for point-to-point, 1 DOF motion. However, many real-world tasks require more DOF and less constrained motion. In a leader-follower dyad, the follower often does not know the end goal when motion begins, or the end goal may change while in motion. In tasks such as these, where the end goal is not definite, point-to-point controllers are not as applicable. Kosuge [5] and Ikeura et al. [6], [7] developed similar strategies for situations that required more flexibility, involving using direction of force and change in magnitude of force. These works sparked work in what is now known as variable-impedance control [8], [9]. This control method offers Cartesian control based on how much the magnitude of the force is changing, and in which direction the force is being applied on the follower. While this model has been shown to be useful for Cartesian movements, [10], [11], it does not generalize to extended objects due to the rotation-translation problem, nor does it address rotational movements alone. Other proposed models for co-manipulation include programming by demonstration (PBD) [12], [13], finite state machines (FSM) [3], [2], uncertainty control [14], and movement coordination [15]. PBD involves some pre-programmed information, and does not easily generalize to a case where a new, unlearned motion is required. Using FSM is promising, but it is necessary to determine how to switch into various states when looking at a $6 \mathrm{DOF}$ controller. Uncertainty control uses uncertainty within a cost function subject to robot dynamics to allow a robot controller to effectively navigate. These techniques have been used in [14], but they are limited to a 2 DOF approach. Movement coordination is another possibility, involving coordinating the movement of a robot with its human partners based on high-level group dynamics, although this method does not take low-level force inputs into account.

\section{B. Intent and Haptic Information}

Human intent is another topic in pHRI. Many papers have suggested that haptic channels are an appropriate method of communication for human intent [16], [17], [18], [19]. This makes sense, as human teams can move objects by interacting only through forces applied to the objects, rather than by communicating verbally or otherwise. Many studies have been done to conclude that robots can be controlled by human force input in this manner, but these studies often involve the human acting directly on the robot, and not through any extended object [20], [21], [7], [22].

Some research involved shared virtual-environment loads [23], [24], and others involved upper arm movements of individuals and dyads [25], [18]. These experiments clarify many aspects of pHRI, including verifying that haptic information aids in co-manipulation tasks, noting some interaction patterns, and combining planning and learning to complete goal-oriented tasks. The virtual-environment loads, however, do not fully capture the details of a human-human dyad physically interacting with one another in a real environment, and using only upper arm movements limits the applications available for a controller based on these movements.

\section{Co-Manipulation Metrics}

Another issue is determining what constitutes a successful controller in co-manipulation. Since haptic information is used as a communication method [26], some researchers have suggested that haptic information is used to minimize a certain criterion. Flash and Hogan [27], for example, 
described human motion as following minimum-jerk trajectories for reaching movements, which has been used to describe motion objectives in many experiments [17], [20]. The theory behind these studies is that robots should move following minimum-jerk trajectories. However, there are also tasks that do not fit well with the minimum-jerk trajectory [28], [1]. Some researchers, like Groten et al. [19], have suggested that the focus should be minimizing the energy of the motion by eliminating interaction forces, or forces that do not contribute to motion. Other examples of metrics include, position error [1], [8], task completion time [10], [28], and wasted effort [17]. It is not clear, however, which of these metrics characterizes the performance of a dyad. For example, a dyad might move slower, giving a higher completion time, but have lower position error. Determining what behavior successful dyads display is essential not only for comparing one dyad to another, but also for comparing one co-manipulation controller to another.

We plan on developing an intuitive controller for comanipulation of extended objects and our approach is to first focus on characterizing human dyad force and motion data through complex co-manipulation tasks. We developed an experiment to this end. The purposes of this study were twofold: first, to provide a baseline for how humans perform a general collaboration task on extended objects, and second, to provide useful haptic information to use for creation of a human intent estimator. Our study provides insights for collaborative motion of dyads not seen in other work, and forms the basis for developing a controller capable of handling complex tasks.

\section{HUMAN DYAD EXPERIMENT}

As a preliminary step in producing a 6-dimensional comanipulation controller between a human and a robot, we performed a study involving human-human teams. If robots are to one day work alongside humans as partners, the robots need to perform tasks in a way that humans intuitively understand the interaction. Our approach is to develop an pHRI controller based on concrete human behavior in order to get performance that humans will understand and find acceptable.

\section{A. Experimental Setup}

After attaining IRB approval, we set up trials involving 2person teams or dyads. These teams were to work together to perform a series of 6 object-manipulation tasks.

1) Table: The object the teams moved was a $59 \times 122 \times 2 \mathrm{~cm}$ wooden board - meant to simulate an object (like a table) that is difficult for one person to maneuver. Attached to the leader end of the board were a pair of ABS 3D-printed handles, to which two ATI Mini45 force/torque sensors were fastened. The sensors transmitted data via ATI NET F/T Net Boxes, which passed data over Ethernet to the computer at a rate of $100 \mathrm{~Hz}$.

The position of the board was tracked via Cortex Motion Capture software with a Motion Analysis Kestrel Digital Realtime System. A total of 8 Kestrel cameras were used to

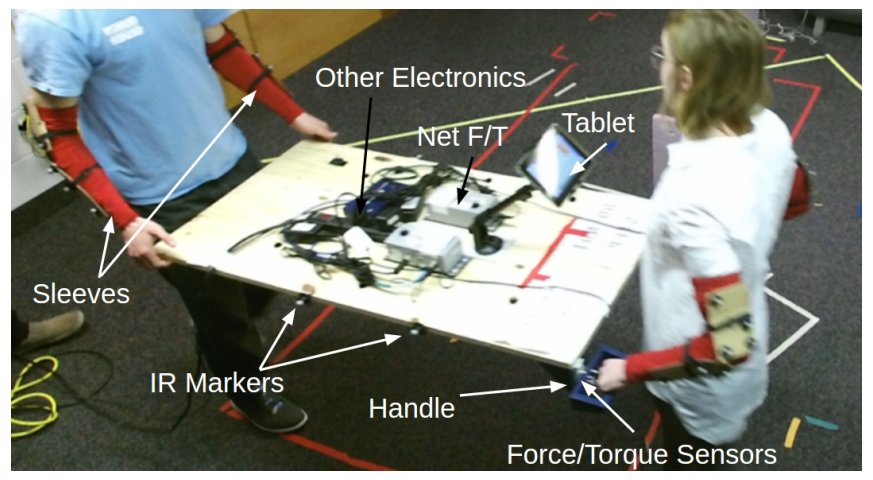

Fig. 2: Setup for table and during trials

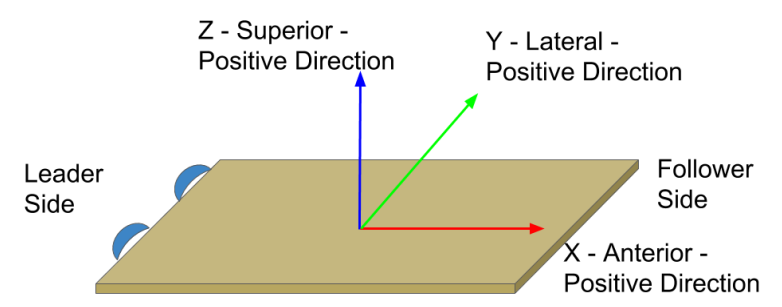

Fig. 3: Anatomical direction reference with corresponding table axis. $\mathrm{X}$ is anterior, $\mathrm{Y}$ is Lateral, and $\mathrm{Z}$ is Superior.

track 8 infrared markers placed on the board. Using a static global frame established by the motion capture system, the position and orientation of the board could be tracked over time, and the force and torque data could be transformed into the board's frame, located at the geometric center of the table (see Fig. 3), as well as the static frame. The motion capture data was collected at a rate of $200 \mathrm{~Hz}$.

Along with the infrared markers and force/torque sensors, the board also held an Ethernet switch, a power strip, and all cables necessary for power and communication. One experimenter was tasked with making sure no obstacles would trip the subjects, including moving these cables as necessary without exerting forces on the table. During the trials, a tablet was mounted on the board to display instructions to the participants. In total, the board weighed $10.3 \mathrm{~kg}$. An annotated visual of the board can be seen in Fig. 2.

2) Subjects: The trial participants were outfitted with polyester arm sleeves for both arms. Two groups of four infrared markers were placed on rigid plates, and then attached to the sleeve, one on the upper arm and one on the lower arm. A blindfold was also used for the tasks where no verbal or visual communication was allowed.

3) Arena: The test arena was a volume measuring 490x510x $250 \mathrm{~cm}$. A series of colored tape lines (see Fig. 4) were placed on the floor of the volume, indicating key positions for each of the 6 object-manipulation tasks. On 3 of the walls surrounding the arena, we placed green, orange, and purple poster boards to help orient the leader when looking at the tablet. As seen in Fig. 5 and Fig. 6, there are colored bars 
on the edges of each task figure representing the walls with the corresponding color. This way, the leader could more easily determine the frame of reference for the instructions on the tablet mounted to the table.

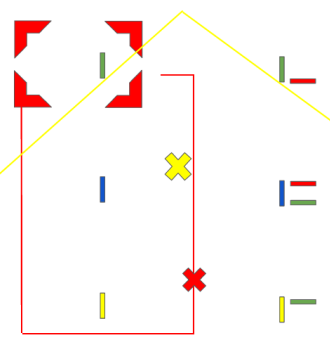

Fig. 4: Colored tape used for task delineation.

The arena was also equipped with a video capturing device. The device we used was a Microsoft Kinect 2, which allowed us to capture 3D point cloud data, as well as color video of each trial. Although we did not need the point cloud data for analysis in this paper, the data may be useful in future work.

\section{B. Experimental Procedure}

First, the participants were oriented on the purpose of the research and signed release forms. Second, a leader was chosen at random (by coin flip). Third, each participant put on the sleeves and the participant designated as the follower placed the blindfold on their head, but not covering their eyes until they were about to perform a blindfolded task. Fourth, two preliminary test runs were performed by the participants with the researchers supervising. These test runs walked the participants through each motion required by the tests - that is translation in $\mathrm{x}, \mathrm{y}$, and $\mathrm{z}$ axes and rotation in $\mathrm{x}, \mathrm{y}$, and $\mathrm{z}$ axes (see Fig. 3 for directions). The first run was done without the follower blindfolded, and the second was with the follower blindfolded. Fifth, the leader then was oriented on following the task instructions via the tablet on the table (see Fig. 5 and Fig. 6). The researchers displayed the task with visual instructions on the tablet, which corresponded to the colored tape on the ground. The leader then followed the instructions as outlined. Sixth, each group ran through the tasks for 1 hour, which allowed each group to run all 6 tasks approximately 6 times each. The tasks were split evenly between blindfolded and nonblindfolded, and were randomized in order for each group of participants. For instance, a group might perform task 1 nonblindfolded, followed by task 4 blindfolded, followed by 3 blindfolded, and so on. We recognized that learning of tasks would occur, but decided the randomization of task order would help to reduce the amount of guessing of the follower, and would encourage a reactive, rather than anticipatory, response. A researcher changed the setup between tasks, and two other researchers ran data collection for motion capture, force/torque, and video. Finally, the participants were debriefed, they filled out a questionnaire about the trials, and were paid.

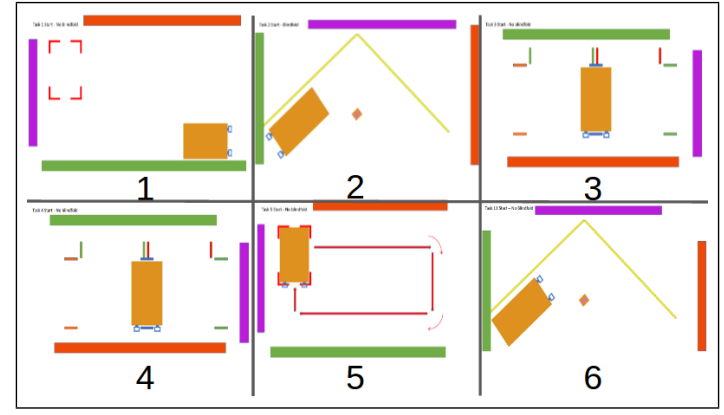

Fig. 5: Starting position for all tasks - Tablet views

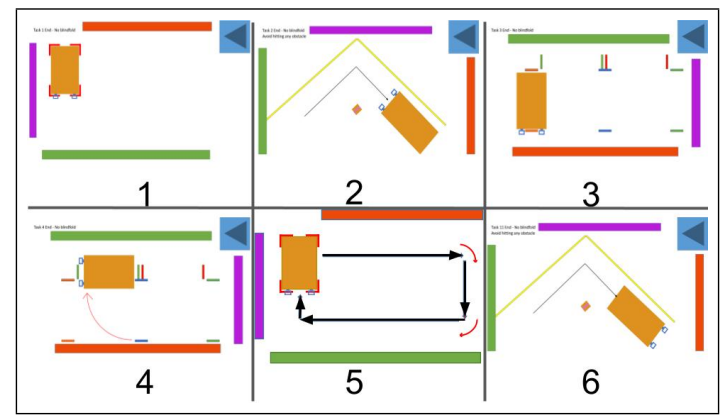

Fig. 6: Ending position for all tasks - Tablet views

The tasks were designed in order to mimic standard motions that humans use when collaborating on moving an object (see Fig. 5 and Fig. 6 for reference), and are outlined as follows:

1) Pick and Place

- Translation and rotation, but emphasizing the location and orientation of object placement

2) Rotation and Translation - Leader facing backwards

- Rotation and translation as needed to navigate trial, meant to simulate a narrow hallway

3) Pure Translation

- Translation in the lateral direction

4) Pure Rotation

- Rotation about the superior axis

5) 3D Complex Task - Translation and Rotation in multiple axes

- Moving object with translation in all three axes while avoiding certain 3D obstacles

6) Rotation and Translation - Leader facing forwards

- Rotation and translation as needed to navigate trial, meant to simulate a narrow hallway

The physical execution of the task started with each participant grasping an end of the board, the leader by the end with sensors and the follower by the end without sensors. They would then lift the table. After which the follower tried to follow the leader as the leader performed the task indicated on the tablet. Once they reached the position, they set the board back on the ground and released. This constituted a single trial. During sighted trials the participants were allowed any method of communication desired. Whereas 
during blind trials the participants were only allowed to communicate via forces applied to the board. A sample of task 5 being performed blindfolded and not blindfolded can be seen at https://youtu.be/i-s1pIs170Y. This task is shown as it encapsulates a majority of the motions seen in all the tasks as they were performed.

\section{Data Collection}

A total of 21 groups participated, and subjects for the trials were recruited using fliers, social media, and word-ofmouth. Trials occurred during February and March of 2016. The participants were comprised of 26 men and 16 women of ages 18-38, and the average age was 22 . There were 38 right-handed and 4 left-handed. A scheduling website was used to facilitate trial sessions, and participants signed up for an available hour-long slot.

If, during a task, any error occurred - such as participants performing a task incorrectly or a failure in data collection - the task was be stopped and repeated.

\section{Data Analysis}

As previously stated, the data acquired for each trial was the force and torque data from the sensors on each handle, the position and orientation of the table, the position and orientation of the participant's arms, as well as the point cloud data from the Kinect 2. The data we were most interested in initially was the force and torque data in relation to the position and orientation of the table. Data from when the blind trials was captured with the objective of characterizing force patterns in human-human dyads that could be used 1) as a baseline for when human-human dyads only use haptic information for co-manipulation and 2) to eventually create a co-manipulation controller that incorporates the force patterns discovered in analysis. Sighted data was captured with the objective of comparing the performance of future comanipulation controllers with an unrestricted human-human dyad.

\section{OBSERVATIONS}

Although the experiment involved 6 different tasks, and our future goals include incorporating controller for $6 \mathrm{DOF}$ translational and rotational movements, this paper focuses on characterizing a subset of the tasks. We focus on the blind versions of task 3 , the pure translation task, as well as some portions of analysis done on task 4, the pure rotation task. The emphasis was placed on these tasks for a few of reasons. First, as discussed in Section II, most research done in this area of pHRI for co-manipulation involved either lateral movement with no extended object, or only anterior direction movements (see Fig. 3 for directions reference). When co-manipulating an extended object, the intent of the leader is complicated by the rotation-translation problem described in Section I. Therefore, characterizing how humans are able to recognize a desired lateral movement with an extended object and distinguish it from a desired rotational movement is key for successful co-manipulation of extended objects. Second, other tasks-such as task 4 and task 5include components of lateral translation. Therefore knowing the defining characteristics of only lateral motion helps to recognize it in more complex tasks. In this paper, we examine only the blind tasks to simplify the analysis, since these tasks involved only haptic communication.

\section{A. Interaction Forces}

Interaction forces are the forces that do not directly relate to motion, i.e. the forces applied by each participant that do not accelerate the object. As suggested by Noohi et al. [17], interaction forces could be used as a source of communication. In our study, the force/torque sensors could not discern between external forces - forces that accelerate the object - and interaction forces, but rather measured the total force applied, so we calculated the interaction force after the experiment ended.

Eq. 1 shows the combined forces that contribute to the total force, or the force measured by the sensors. $F_{t}$ is the total force on the object, $F_{i}$ is the interaction force, and $F_{e}$ is the external force. The motion capture data described the pose of the table over time, and was differentiated twice to acquire the acceleration data. With a known mass of the table and acceleration, the external force was estimated (Eq. 2), and removed from the total force to give us the interaction force for the task.

$$
\begin{gathered}
F_{t}=F_{i}+F_{e} \\
F_{e}=m a
\end{gathered}
$$

For the anterior, $X$, and lateral, $Y$, directions, the only external force being applied is the force applied from the participants, whereas in the vertical $Z$ direction, gravity was also applied. For all calculations and analysis in this paper, the forces were low-pass filtered near $20 \mathrm{~Hz}$ to represent human response ranges. The muscle response of humans can reach up to $100 \mathrm{~Hz}$ for brief, forceful efforts, but often lies within the 10-30 $\mathrm{Hz}$ range [29].

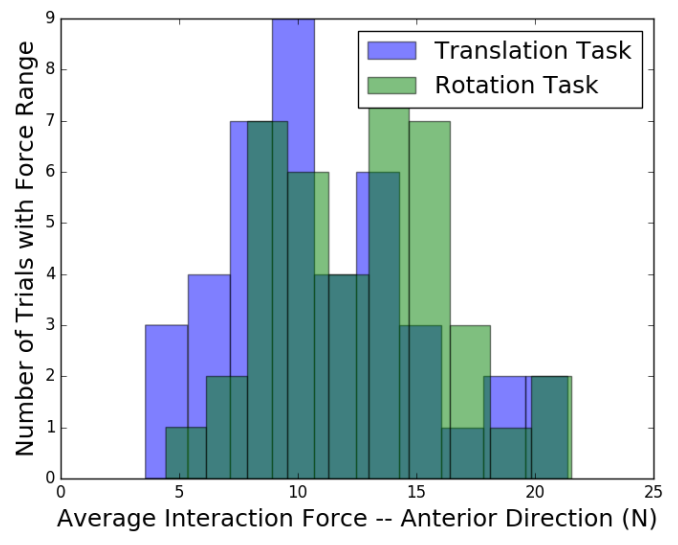

Fig. 7: Histogram of lateral movement average force along anterior-lateral plane 


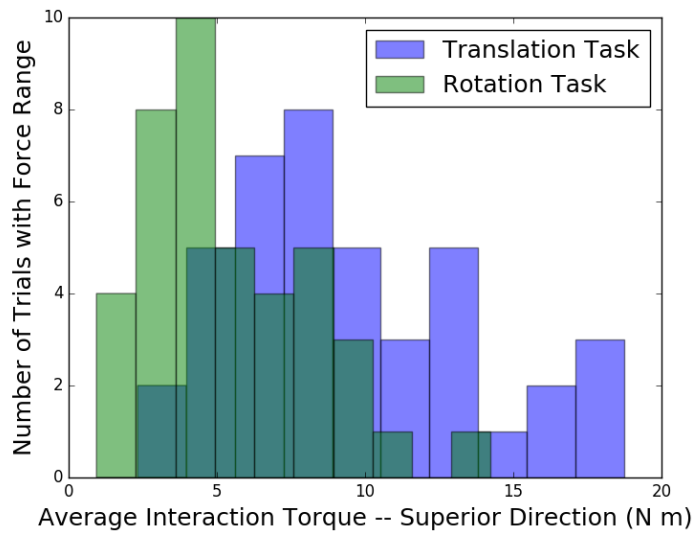

Fig. 8: Histogram of lateral movement average interaction torque about superior axis

As mentioned in Section II, some prior work in pHRI has presented an objective of pHRI controllers as minimizing interaction forces by driving them to zero magnitude [19]. In fact, this is also a characteristic of variable-impedance control [10], [8]. Our study, however, showed that this may not always be the case. For both lateral and rotational movements, we calculated the average interaction force in the anterior direction and the average interaction torque about the superior direction. We considered both task 3 and task 4 in order to determine if the interaction forces were minimized for one task but not for another, as well as to identify unique characteristics between the two similar tasks. Histograms showing the distribution of average force and torque over the duration of the trial are shown in Fig. 7 and 8. As can be seen, the interaction force was almost always non-zero for both lateral and rotational movements in the anterior direction (see Fig. 3 for clarity on directions). Additionally, we considered the ratio of average interaction to external forces, $F_{i, a v g} / F_{e, \text { avg }}$, which-when averaged over all the trials-gave a magnitude of 20 , indicating the forces used for acceleration of the object were 20 times smaller than those not used for acceleration. It is not clear why the average interaction force was so substantial, but our hypotheses include:

1) These forces were used for object and human stability

2) These forces were used to communicate intent

We will conduct future studies with respect to the stability hypothesis, but the intent hypothesis is discussed further in Section IV-C. This result is significant because it implies that lateral collaborative movements rely on interaction forces along the anterior direction, which is not seen in many state-of-the-art pHRI controllers. Another takeaway is that minimizing interaction forces may not yield results easily understood by human partners in co-manipulation tasks, since it is now evident that humans are not necessarily minimizing these forces. We also notice from Fig. 8 that the average torque about the superior axis is generally greater in magnitude for translation tasks than it is for rotation task, which may be one indicator of how human-human dyads solve the rotation-translation ambiguity problem.

\section{B. Minimum-Jerk}

The minimum-jerk (MJ) movement is well-documented as a basis for human arm movements, especially in pointto-point movements. However, we did not expect to see MJ trajectories in these trials, since one participant was blindfolded and unaware of the task specifications, and the dyads used whole-body motion rather than arm-only motion. However, another interesting finding from our study was that the lateral movement tasks resembled a MJ movement in the lateral direction, especially for the dyads that completed the task more quickly. Fig. 9 shows the correlation between deviation from MJ trajectories and an increase in time to complete the task. The slower dyads often had a larger error between their position, and the ideal MJ position, whereas the quicker dyads generally had a smaller error with respect to a MJ trajectory.

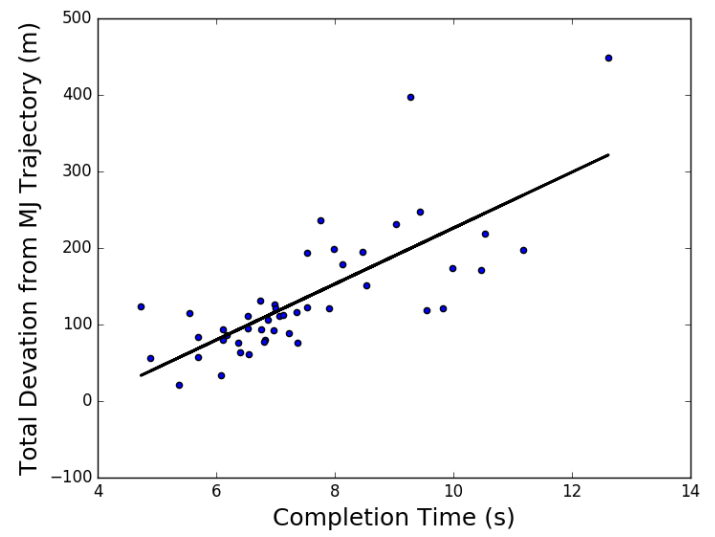

Fig. 9: Comparing completion time to deviation from MJ trajectory

Overall, the lateral movement stayed close to the MJ trajectory, and adhering to a similar trajectory over all trials corroborates the results of similar 1-dimensional study [3]. Fig. 10 shows the lateral position of the lateral tasks over time. The gray dotted lines show each individual task, the black dotted line is the average of all the tasks, and the blue line is the ideal MJ trajectory given an average start and stop position. As we can see, even though the follower did not know the end position, they stayed fairly close to the MJ trajectory in the lateral direction. Although, there were some trials that did not adhere closely to a MJ trajectory due to disturbances, or disagreement in intent. 


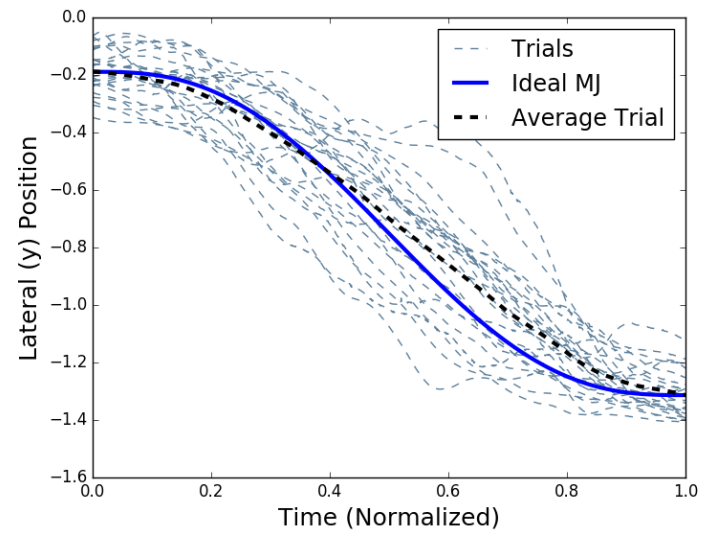

Fig. 10: Lateral trial trajectory with ideal MJ and average trajectories

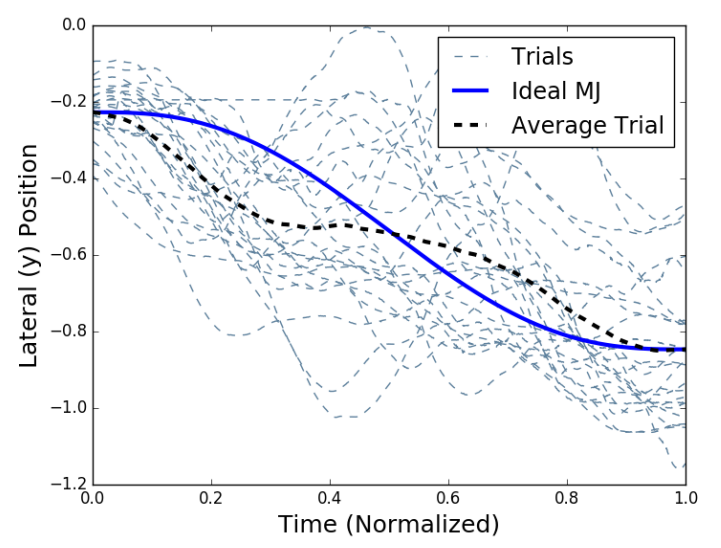

Fig. 11: Rotational trial trajectory with ideal MJ and average trajectories

However, comparing the translation task with the rotation task, see Fig. 11, shows that achieving MJ trajectories may not always be the goal of the dyads during coordinated motion. As we see, the average trajectory in Fig. 11 was significantly distinct from the MJ trajectory, and looking at each individual trial shows that there were a variety of dissimilar paths taken in the rotation.

Our conclusion to this point agrees with previous research that suggests MJ trajectories are not useful as a basis for control as it is too restrictive [1] and does not extend to rotation tasks. We also conclude that $\mathrm{MJ}$ trajectories can be useful for describing translation task metrics (discussed more in Section IV-D)

\section{Lateral Movement Start Characteristics}

In the case of lateral movements, we recognized some patterns in how the dyads behaved. Studying the videos of the lateral motion task, we saw that the follower often guessed the leader's intent wrong, and began to rotate when the leader started their movement. When this happened, the leader would flex their arm on one side of the table, causing a torque on the table, and the follower would then commence moving in the correct manner. Upon seeing this, we started to look for a pattern of applied torques that would indicate the start of a lateral movement.

Fig. 13 illustrates the pattern we found for lateral motion with an extended object. The leader applied opposing forces with each arm, which caused an increase in magnitude of the interaction torque about the $Z$ axis. The increasing magnitude of the slope, or time derivative of torque, signaled to the follower that the leader wanted to move laterally. After the torque threshold was met, the follower moved and the desired lateral movement began. We then searched through each trial for the first instance of meeting the torque threshold and noted the trigger time. We then determined whether the pattern held based on whether the table's $Y$ velocity at the trigger time matched the first instance of movement in the lateral direction for the trial, as shown in Fig. 12. This method was capable of correctly predicting the start time for $75 \%$ (35 of 46) of all blind, translation trials with useable data. It is not clear how the velocity is affected by the forces after this point, but this is an important open research question that we are still exploring in order to make a robot effective for co-manipulation.

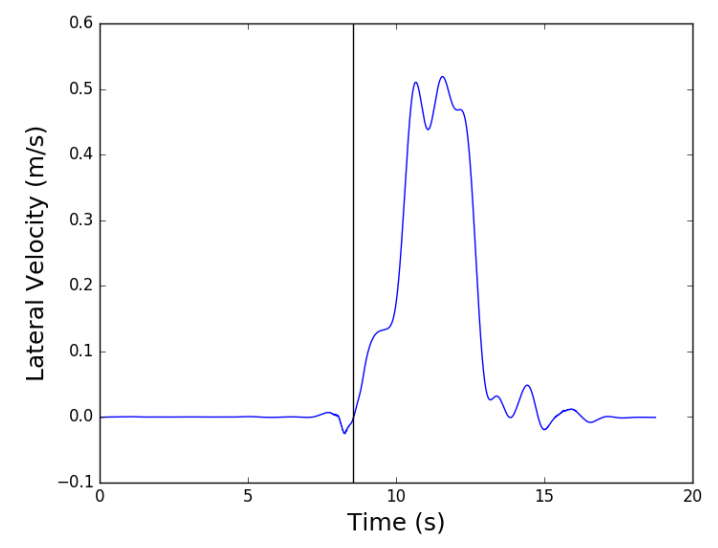

Fig. 12: Velocity (lateral direction) with line delineating start point

Characterizing the force pattern causing a lateral movement is an important development in human-robot comanipulation. Distinguishing between rotation and translation was difficult even for some of the participants of our trials. By determining what humans do to cause these movements, we can develop methods of communicating to the robot human intent more accurately than with previous methods.

\section{Metric Observations}

Our objective for future work is to develop control algorithms for robots to successfully co-manipulate extended objects in a human-robot dyad. We have begun some initial work on human intention estimation from force data and a demonstration of simple pHRI [30]. However, to classify the performance of such a controller in comparison with humanhuman dyads, we need to first quantify the performance of 


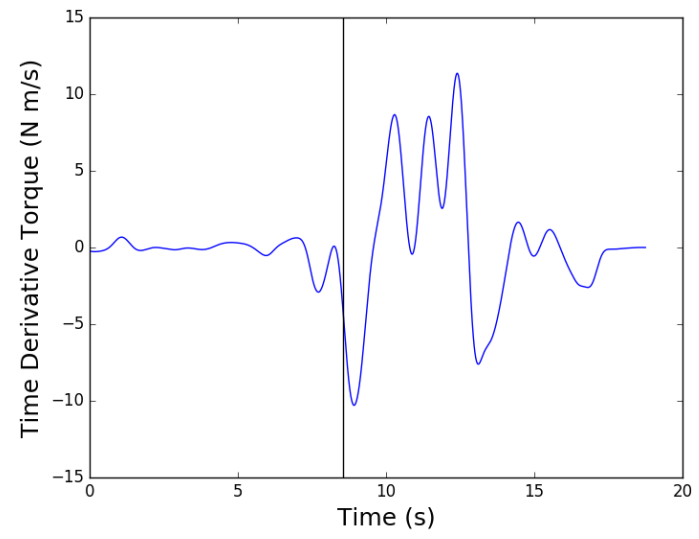

Fig. 13: Trigger pattern (anterior direction) for time derivative of torque about $Z$ axis with line delineating start point

the human-human dyads. Therefore, we needed to define which metrics would indicate how well a certain dyad performed a certain trial. This is difficult since all subjects were able to complete the trials, and were given no specific directions other than for the leader to complete the onscreen objectives, and the follower to follow the leader. However, from observing the trials, it was clear that some dyads performed the tasks in a smooth, coordinated, and timely manner, and we sought to discover what metrics characterized the performance of the best dyads. Possible metrics considered were:

- Task Completion Time

- Distance Covered by Table

- Average/Max Force on Table

- Average/Max Velocity of Table

- Average/Max Power of Table

- Average/Max Angular Velocity of Table

- Deviation from MJ Trajectory of Table

We expected there to be some correlation between some of these measurements since most of the metrics were proposed in previous research [1] [28] [31]. The metrics were compared using the Pearson correlation coefficient, and surprisingly offered very little correlation. For the lateral task, the metrics that were most applicable were average/max angular velocity and deviation from the MJ trajectory. We expected a good task to be one where the table minimized the average angular velocity about the $z$ axis, and stayed relatively close to the MJ trajectory, but the correlation coefficient between these two metrics was 0.05 . In fact, there was a much stronger correlation between deviation from MJ trajectory and completion time, average lateral velocity, and distance covered - being $0.63,-0.42$, and 0.38 respectively. Intuitively, minimizing angular velocity would be an ideal metric for this task, but the fact that deviation from MJ trajectory corresponds so well with the other metrics means it should be a point of emphasis in future research. Although these observations provide some insight to this topic, our work on determining metrics is still an open question that is necessary to quantify performance of human-robot comanipulation.

\section{CONCLUSION AND Future Work}

In this paper, we have discussed the problems and limitations of many current co-manipulation pHRI controllers. We discussed the advantages of creating control methods based on human-human dyad behavior to increase the ability of human-robot dyads to adapt to less-defined situations. We also described our experiment gathering the force and motion data for several simple and complex tasks involving human dyads. The main takeaways from this data are that interaction forces play an important role in communicating intent between dyads in co-manipulation and that they are likely not minimized as previously supposed. Lateral movements display characteristics of minimum-jerk movements in the lateral direction.

Our future work includes characterizing the rotationtranslation problem more fully by determining start and stop triggers for both cases. We will then create a co-manipulation controller for general planar motion of an extended object. The controller will be compared with the performance of both the blind and sighted dyads from our study, providing a lower and upper bound, respectively, for the performance of our controller. After completing the planar controller, we will extend our work to include the other tasks and creating a 6 DOF controller for co-manipulation of an extended object. To our knowledge, no 6 DOF pHRI co-manipulation controller based on human-human dyad behavior exists. Although future work may also require including additional sensory cues and information (e.g. auditory or visual), we expect that the data and observations presented in this paper are a solid foundation to allowing humans and robots to intuitively interact for general co-manipulation tasks.

\section{ACKNOWLEDGEMENTS}

This work is supported by the Army RCTA program. All results and conclusions are the responsibility of the authors and do not necessarily reflect the opinions of the funding source.

\section{REFERENCES}

[1] A. Thobbi, Y. Gu, and W. Sheng, "Using human motion estimation for human-robot cooperative manipulation," IEEE International Conference on Intelligent Robots and Systems, pp. 2873-2878, 2011.

[2] A. Bussy, P. Gergondet, A. Kheddar, F. Keith, and A. Crosnier, "Proactive behavior of a humanoid robot in a haptic transportation task with a human partner," Proceedings - IEEE International Workshop on Robot and Human Interactive Communication, no. 2, pp. 962-967, 2012.

[3] A. Bussy, A. Kheddar, A. Crosnier, and F. Keith, "Human-humanoid haptic joint object transportation case study," IEEE International Conference on Intelligent Robots and Systems, pp. 3633-3638, 2012.

[4] Y. Karayiannidis, C. Smith, and D. Kragic, "Mapping human intentions to robot motions via physical interaction through a jointly-held object," Proceedings - IEEE International Workshop on Robot and Human Interactive Communication, vol. 2014-Octob, no. October, pp. 391-397, 2014.

[5] K. Kosuge, H. Yoshida, and T. Fukuda, "Dynamic control for robothuman collaboration," Proceedings of 1993 2nd IEEE International Workshop on Robot and Human Communication, pp. 398-401, 1993. [Online]. Available: http://ieeexplore.ieee.org/document/367685/ 
[6] R. Ikeura, H. Monden, and H. Inooka, "Cooperative Motion Control of a Robot and a Human,” pp. 2-3, 2002.

[7] M. M. Rahman, R. Ikeura, and K. Mizutani, "Impedance characteristic of human arm for cooperative robot," pp. 1455-1460, 2002.

[8] R. Ikeura and H. Inooka, "Variable Impedance Control of a Robot for Cooperation with a Human," pp. 3097-3102, 1995.

[9] F. Dimeas and N. Aspragathos, "Reinforcement learning of variable admittance control for human-robot co-manipulation," IEEE International Conference on Intelligent Robots and Systems, vol. 2015Decem, pp. 1011-1016, 2015.

[10] V. Duchaine and C. M. Gosselin, "General model of human-robot cooperation using a novel velocity based variable impedance control," Proceedings - Second Joint EuroHaptics Conference and Symposium on Haptic Interfaces for Virtual Environment and Teleoperator Systems, World Haptics 2007, pp. 445-451, 2007.

[11] F. Ficuciello, L. Villani, and B. Siciliano, "Variable Impedance Control of Redundant Manipulators for Intuitive Human-Robot Physical Interaction," IEEE Transactions on Robotics, vol. 31, no. 4, pp. 850-863, 2015.

[12] E. Gribovskaya, A. Kheddar, and A. Billard, "Motion learning and adaptive impedance for robot control during physical interaction with humans," Proceedings - IEEE International Conference on Robotics and Automation, pp. 4326-4332, 2011.

[13] L. Rozo, S. Calinon, D. G. Caldwell, P. Jimenez, and C. Torras, "Learning Physical Collaborative Robot Behaviors From Human Demonstrations," IEEE Transactions on Robotics, vol. 32, no. 3, pp. 513-527, 2016.

[14] J. R. Medina, T. Lorenz, and S. Hirche, "Synthesizing anticipatory haptic assistance considering human behavior uncertainty," IEEE Transactions on Robotics, vol. 31, no. 1, pp. 180-190, 2015.

[15] L. D. Riek and S. Member, "Movement Coordination in Human Robot Teams :," vol. 32, no. 4, pp. 909-919, 2016

[16] C. Basdogan, C.-h. Ho, M. A. Srinivasan, and M. E. L. Slater, "An Experimental Study on the Role of Touch in Shared Virtual Environments," vol. 7, no. 4, pp. 443-460, 2001.

[17] E. Noohi, M. Zefran, and J. L. Patton, "A Model for Human-Human Collaborative Object Manipulation and Its Application to HumanRobot Interaction," IEEE Transactions on Robotics, vol. 32, no. 4, pp. 880-896, 2016.

[18] K. B. Reed, J. Patton, and M. Peshkin, "Replicating Human-Human Physical Interaction," no. April, pp. 10-14, 2007.

[19] R. Groten, D. Feth, R. L. Klatzky, and A. Peer, "The role of haptic feedback for the integration of intentions in shared task execution," IEEE Transactions on Haptics, vol. 6, no. 1, pp. 94-105, 2013.

[20] B. Corteville, E. Aertbelien, H. Bruyninckx, J. De Schutter, and H. Van Brussel, "Human-inspired robot assistant for fast point-topoint movements," Proceedings - IEEE International Conference on Robotics and Automation, no. April, pp. 3639-3644, 2007.

[21] R. Ikeura, A. Morita, and K. Mizutani, "Variable damping characteristics in carrying an object by two humans," Proceedings 6th IEEE International Workshop on Robot and Human Communication. ROMAN'97 SENDAI, pp. 130-134, 1997.

[22] T. Tsumugiwa, R. Yokogawa, and K. Ham, "Variable Impedance Control Based on Estimation of Human Arm Stiffness for HumanRobot Cooperative Calligraphic Task," no. May, pp. 644-650, 2002.

[23] C. E. Madan, A. Kucukyilmaz, T. M. Sezgin, and C. Basdogan, "Recognition of Haptic Interaction Patterns in Dyadic Joint Object Manipulation," IEEE Transactions on Haptics, vol. 8, no. 1, pp. 5466, 2015.

[24] M. Lawitzky, J. R. Medina, D. Lee, and S. Hirche, "Feedback motion planning and learning from demonstration in physical robotic assistance: Differences and synergies," IEEE International Conference on Intelligent Robots and Systems, pp. 3646-3652, 2012.

[25] R. P. R. D. V. D. Wel, G. Knoblich, and N. Sebanz, "Let the Force Be With Us : Dyads Exploit Haptic Coupling for Coordination," vol. 37, no. 5, pp. 1420-1431, 2011.

[26] A. Sawers, T. Bhattacharjee, J. L. McKay, M. E. Hackney, C. C. Kemp, and L. H. Ting, "Small forces that differ with prior motor experience can communicate movement goals during human-human physical interaction,' Journal of NeuroEngineering and Rehabilitation, vol. 14, no. 1, p. 8, 2017. [Online]. Available: http://jneuroengrehab. biomedcentral.com/articles/10.1186/s12984-017-0217-2

[27] T. Flash and N. Hogan, "The coordination of arm movements: an experimentally confirmed mathematical model." The Journal of neuroscience, vol. 5, no. 7, pp. 1688-1703, 1985. [Online]. Available: http://www.ncbi.nlm.nih.gov/pubmed/4020415

[28] S. Miossec and A. Kheddar, "Human motion in cooperative tasks: Moving object case study," 2008 IEEE International Conference on Robotics and Biomimetics, ROBIO 2008, no. i, pp. 1509-1514, 2008.

[29] D. W. F. Etienne Burdet and T. E. Milner, Human Robotics: Neuromechanics and Motor Control. 55 Hayward Street, Cambridge, MA 02142: Massachusetts Institute of Technology, 2013.

[30] E. C. Townsend, "Estimating Short-Term Human Intent for Physical Human-Robot Co-Manipulation," Master's thesis, Brigham Young University, 2017.

[31] R. K. Groten, "Haptic Human-Robot Collaboration : How to Learn from Human Dyads," 2011. 